\title{
The direction of the difference between Canadian and American erythrocyte folate concentrations is dependent on the assay method employed: a comparison of the Canadian Health Measures Survey and National Health and Nutrition Examination Survey
}

\author{
Cynthia K. Colapinto ${ }^{1,2}$, Mark S. Tremblay ${ }^{1,3}$, Susanne Aufreiter ${ }^{4}$, Tracey Bushnik ${ }^{5}$, Christine M. Pfeiffer ${ }^{6}$ \\ and Deborah L. O'Connor ${ }^{4,7 *}$ \\ ${ }^{1}$ Healthy Active Living and Obesity Research Group, Children's Hospital of Eastern Ontario Research Institute, \\ Ottawa, ON, Canada \\ ${ }^{2}$ Institute of Population Health, University of Ottawa, Ottawa, ON, Canada \\ ${ }^{3}$ Department of Pediatrics, University of Ottawa, Ottawa, ON, Canada \\ ${ }^{4}$ Physiology and Experimental Medicine Program, The Hospital for Sick Children, Toronto, ON, Canada \\ ${ }^{5}$ Health Analysis Division, Statistics Canada, Ottawa, ON, Canada \\ ${ }^{6}$ National Center for Environmental Health, Centers for Disease Control and Prevention, Atlanta, GA, USA \\ ${ }^{7}$ Department of Nutritional Sciences, University of Toronto, Toronto, ON, Canada
}

(Submitted 22 April 2014 - Final revision received 25 June 2014 - Accepted 19 August 2014 - First published online 8 October 2014)

\section{Abstract}

Fortification of select grain products with folic acid and periconceptional supplementation recommendations in Canada and the USA have improved folate status, and have been associated with a reduced risk of neural tube defects. In the present study, we aimed to conduct a comparison of erythrocyte folate concentrations from the 2007-9 Canadian Health Measures Survey (CHMS) and the 2007-8 US National Health and Nutrition Examination Survey (NHANES). Erythrocyte folate concentration was assessed in participants aged 6-79 years (CHMS, $n$ 5248; NHANES, $n$ 7070). To account for different folate assays employed - Immulite 2000 immunoassay (CHMS) and microbiological assay (NHANES) - a conversion equation was generated ( $n 152$ adults) to adjust the CHMS data. $t$ Tests were used to examine country differences. Median Canadian erythrocyte folate concentrations (method-adjusted) were lower than those of Americans (988 and $1100 \mathrm{nmol} / \mathrm{l}$, respectively), but unadjusted median Canadian erythrocyte folate concentrations were higher (1250 nmol/l). The upper 95\% CI boundary of the method-adjusted Canadian erythrocyte folate distribution overlapped that of the American erythrocyte folate concentrations, while the lower 95\% CI boundary of the method-adjusted Canadian erythrocyte folate data was below the American distribution. In summary, the fact that erythrocyte folate concentrations were either higher or lower in Canadians compared with Americans, depending on whether an adjustment was made to account for assay differences, suggests that caution must be exercised in evaluating erythrocyte folate data from different countries because analytical methods are not readily comparable. Furthermore, we cannot unequivocally conclude that there are true differences in erythrocyte folate concentrations between the Canadian and American populations in the post-fortification era

Key words: Folic acid: Erythrocyte folate: Folate status: National Health and Nutrition Examination Survey: Canadian Health Measures Survey

Fortification of white wheat flour, and other select grains, with folic acid and periconceptional supplementation guidelines are designed to improve folate status, and have been associated with a reduced risk of neural tube birth defects (NTD). The USA and Canada were among the first countries to fully implement mandatory policies specific to the fortification of certain grain products with folic acid in $1998^{(1,2)}$. The US
Food and Drug Administration requires folic acid fortification of all enriched cereal grain products - including wheat flour, maize grits, maize meal, rice and pasta - at levels of $0.95-309 \mathrm{mg} / 100 \mathrm{~g}$, based on a fortification level of $0.14 \mathrm{mg} /$ $100 \mathrm{~g}$ of the cereal grain product. These ranges are intended, in part, to account for cooking losses in some products. In Canada, white wheat flour and enriched maize meal are

Abbreviations: CHMS, Canadian Health Measures Survey; MAPE, mean absolute percentage error; NHANES, National Health and Nutrition Examination Survey; NTD, neural tube birth defects.

*Corresponding author: D. L. O'Connor, email deborah_l.oconnor@sickkids.ca 
fortified with folic acid at a level of $0 \cdot 15 \mathrm{mg} / 100 \mathrm{~g}$, and enriched pasta is fortified at a level of $0.20 \mathrm{mg} / 100 \mathrm{~g}^{(2)}$. Discretionary fortification of ready-to-eat breakfast cereals with folic acid allows up to $0.40 \mathrm{mg} /$ serving in the USA, and up to $0.06 \mathrm{mg} /$ serving in Canada. Monitoring the effectiveness and safety of an intervention should accompany any change in population-level policy to assess the need for modification. Folic acid intake in pregnancy has been associated with a significant reduction in NTD ${ }^{(3-5)}$. Although folic acid policies are somewhat different between Canada and the USA, these have been credited with a reduction in NTD rates since the programmes were implemented ${ }^{(6-8)}$. Currently, more than fifty countries have reported a requirement to add folic acid to flour; however, regulations may not be implemented in all countries $^{(9,10)}$.

In addition to public policy regarding food fortification, the USA has adopted the Institute of Medicine guidelines recommending that women of childbearing age consume $0.40 \mathrm{mg} / \mathrm{d}$ of folic acid from fortified foods, supplements or both in addition to natural food sources of folate to reduce the risk of $\mathrm{NTD}^{(3,11)}$. In Canada, this amount is recommended in the form of a multivitamin supplement, in addition to folate-rich foods ${ }^{(12)}$

Erythrocyte folate concentrations have been monitored regularly in the USA through the National Health and Nutrition Examination Survey (NHANES) since $1976^{(13)}$; however, erythrocyte folate concentrations of Canadians at a national level have only recently been examined through the Canadian Health Measures Survey (CHMS) ${ }^{(14)}$. Virtually no clinical folate deficiency (erythrocyte folate concentration $<305 \mathrm{nmol} / 1$ ) has been reported in these population-level surveys in either country since folic acid fortification of the food supply, though concerns have now shifted towards the higher erythrocyte folate concentrations that have been observed ${ }^{(14,15)}$. Still, some women of childbearing age may not be achieving erythrocyte folate concentrations considered optimal for NTD risk reduction ${ }^{(14,16)}$. Furthermore, there has never been a national-level comparison of folate concentrations between Canada and the USA. Such a comparison is complicated as two different assay methods were used for measuring erythrocyte folate concentration - the 2007-9 CHMS used the Immulite 2000 immunoassay (Siemens Canada Limited) and the 2007-8 NHANES used a microbiological assay. It is well known that blood folate concentrations vary depending on the laboratory in which they are conducted and the assay employed $^{(17-20)}$. In one study in the literature, the Immulite 2000 immunoassay (Siemens Canada Limited) was found to yield higher concentrations of erythrocyte folate than the microbiological assay ${ }^{(21)}$. Whether the direction of this difference was specific to this laboratory or, in fact, reflects an assay-dependent bias is not clear. Considering both the similarities and important differences in Canadian and American folic acid fortification and supplementation policies, the aim of the present study was to compare erythrocyte folate concentrations in the Canadian and American populations. This comparison was facilitated by generating a conversion equation through a method-comparison experiment.

\section{Methods}

The analyses included data from the 2007-9 CHMS and the 2007-8 NHANES. The methodology for each survey is described briefly herein, and has been explained in greater detail elsewhere ${ }^{(22,23)}$.

\section{Sampling and survey methods}

Canadian Health Measures Survey. The CHMS used a complex, multi-stage, cluster sampling protocol to achieve a nationally representative cross-sectional sample. The final sample included 5604 Canadians aged 6-79 years balanced by sex in each of the following age groups: 6-11, 12-19, 20-39, 40-59 and 60-79 years. This sample was representative of approximately $96 \%$ of the Canadian population. An interviewer administered a detailed health questionnaire, in each participant's home, which included questions on sociodemographic characteristics and dietary supplement use. A certified phlebotomist collected blood samples $1 \mathrm{~d}$ to 6 weeks later in a Mobile Examination Centre to measure a variety of analytes, including erythrocyte folate ${ }^{(22,24)}$. The Health Canada Research Ethics Board approved the CHMS protocol and the Children's Hospital of Eastern Ontario and University of Ottawa Research Ethics Boards approved the secondary data analysis.

National Health and Nutrition Examination Survey. The 2007-8 NHANES is a 2-year cycle release from the continuous NHANES survey. This survey employed a complex, multistage design and is representative of the civilian, noninstitutionalised US population ${ }^{(23)}$. This research included a final sample of 6- to 79-years-olds ( $n$ 7996), from a total sample of 10149 participants, to match the age range for the CHMS. Similar to the CHMS, participants completed an in-home interviewer-administered survey, which included information on sociodemographic characteristics and dietary supplement use, followed by a physical examination and blood collection in a Mobile Examination Centre 1-2 weeks later. The NHANES protocol was reviewed and approved by the National Center for Health Statistics (NCHS) Research Ethics Review Board.

Erythrocyte folate concentrations. A total of 5248 CHMS participants and 7070 NHANES participants had erythrocyte folate data available.

Erythrocyte folate allows for an estimate of tissue folate stores and is therefore considered a long-term indicator of status $^{(25)}$. The CHMS and NHANES collected venepuncture samples in EDTA-treated vacutainers that were immediately processed on-site. The NHANES also collected regular redcapped vacutainers for serum collection. After haematocrit measurement, aliquots of whole blood were frozen, stored at $-20^{\circ} \mathrm{C}$ and shipped weekly on dry ice to the Health Canada Nutrition Laboratory (CHMS) or the Centers for Disease Control and Prevention Laboratory (NHANES) ${ }^{(22,26)}$. The NHANES diluted whole blood (1:11) with $10 \mathrm{~g} / \mathrm{l}$ of ascorbic acid solution before freezing. The NHANES also generated serum stabilised with $0.5 \%(\mathrm{w} / \mathrm{v})$ sodium ascorbate, for later determination of folate content. 
Canadian Health Measures Survey immunoassay. Researchers thawed and then diluted whole-blood samples (1:26) with $5 \mathrm{~g} / 1$ of ascorbic acid solution, allowed them to incubate for $180 \mathrm{~min}$ at room temperature, and then analysed the samples for folate using the Immulite 2000 immunoassay (Siemens Canada Limited) $^{(27)}$. Researchers calculated erythrocyte folate concentration from the measured whole-blood folate concentration, adjusting for erythrocyte volume but without correction for serum folate concentration. The CHMS laboratory assessed the accuracy and reproducibility of the whole-blood folate assay using the manufacturers' serum controls (Con6: Tri-level multi-constituent control) and whole-blood controls (Bio-Rad Lyphochek Trilevel; Bio-Rad Laboratories). These controls had an inter-assay $\mathrm{CV}$ of $6-8.5 \%$ for serum and $8 \%$ for whole blood. All the analysed controls (serum and whole blood) were within $10 \%$ of target values.

National Health and Nutrition Examination Survey microbiological assay. The NHANES laboratory thawed and then prepared samples by diluting serum (1:100) and wholeblood lysates (1:140) with $5 \mathrm{~g} / 1$ of sodium ascorbate ${ }^{(20,26)}$. These dilution factors are appropriate for a population consuming folic acid-fortified foods. Researchers added diluted samples to a ninety-six-well plate together with assay medium containing chloramphenicol-resistant Lactobacillus rhamnosus (American Type Culture Collection no. 27773; National Collection of Industrial Bacteria 10463) and all of the nutrients necessary for the growth of $L$. rhamnosus, with the exception of folate, and then incubated for $45 \mathrm{~h}$ at $37^{\circ} \mathrm{C}$. The calibration curve for this assay was generated using 5 -methyltetrahydrofolate from Merck \& $\mathrm{Cie}^{(20,26)}$. The total folate concentration was assessed by measuring the turbidity of the inoculated medium at $590 \mathrm{~nm}$ in a PowerWave microplate reader (Bio-Tek Instrument). Researchers calculated erythrocyte folate concentration from the measured whole-blood folate concentration, adjusting for erythrocyte volume and correcting for serum folate concentration ${ }^{(28)}$. Long-term $\mathrm{CV}$ from three whole-blood lysate quality-control pools analysed in every run were $8 \cdot 0-14 \%$ at $402-1570 \mathrm{nmol} / \mathrm{l}$. The National Institute for Biological Standards and Control WHO reference material (lyophilised) for whole-blood folate (95/528) was analysed several times a year to verify comparability of the results over time. The mean value obtained was 11.5 (SD 1.15) ng/ ampoule compared with an assigned value of $13 \mathrm{ng} / \mathrm{ampoule}$.

Cut-offs. The only formally accepted cut-offs associated with erythrocyte folate status are those associated with deficiency, which are based on haematological indicators (e.g. macrocytic anaemia) as well as metabolic indicators (e.g. folate concentration below which plasma homocysteine concentrations start to rise $)^{(11,29)}$. We used the classical definition of erythrocyte folate deficiency $<305 \mathrm{nmol} / 1 \quad(<140 \mathrm{ng}$ / $\mathrm{ml}$ ). This cut-off was defined by the Institute of Medicine based on several studies demonstrating the appearance of hypersegmented neutrophils, a characteristic of megaloblastic anaemia, at erythrocyte folate concentration below this level ${ }^{(11)}$.

Selected sociodemographic factors of interest. Both surveys used self-reported sociodemographic characteristics. For the present study, age groups of the CHMS sample were used. We examined socio-economic status by per-person household income equivalents (which grouped respondents into quintiles after adjusting for family size and composition) for each country separately. We defined race/ethnicity as nonHispanic white or 'other' and assigned missing responses for race/ethnicity to the largest parameter (non-Hispanic white) since the percentage of missing responses was small $(<3 \%)$.

Supplement use. CHMS interviewers collected drug identification and natural health product numbers from containers shared by participants at the household visit. Researchers verified this information at the clinic visit and collected information about any changes in drug and supplement usage at this time ${ }^{(30)}$. We determined supplemental folic acid use - whether consumed alone or as a multivitamin - in the $30 \mathrm{~d}$ before the clinic visit by matching drug identification and natural health product numbers with product information extracted from the Health Canada Drug Product and Licensed Natural Health Product databases ${ }^{(31,32)}$. We used the derived variable in the NHANES public data file for use of folic acidcontaining supplements in the last $30 \mathrm{~d}$. NHANES participants showed the supplement container at the household interview, or if this was unavailable, named the supplement verbally. Trained nutritionists reviewed these data and matched entries with the in-house NCHS Product Label Database. If no match was available, the product was added or a product of similar nutrient composition was imputed.

\section{Cross-over equation sub-study}

We conducted an erythrocyte folate method-comparison study to generate a conversion equation between the immunoassay employed in the CHMS and the microbiological assay used by the NHANES. A convenience sample of 152 healthy men and women, aged 16-79 years, was recruited from The Hospital for Sick Children (SickKids) and the greater Toronto area. Participants attended the Clinical Investigation Unit at SickKids where written informed consent was secured and then blood was drawn. Following determination of haematocrit, aliquots of the whole-blood sample were immediately frozen (Immulite 2000 immunoassay; Siemens Canada Limited) or diluted with a $1: 11$ solution of $10 \mathrm{~g}$ ascorbic acid/1 (microbiological assay). Folate concentrations were determined by the Immulite 2000 immunoassay (Siemens Canada Limited) at the Health Canada CHMS laboratory using the methods described above and by the microbiological assay at SickKids. The manufacturer's kits used for the immunoassay were the same as those used for the CHMS, though the short shelf-life of the kits led to the use of several different lots. The manufacturer did not release any notices regarding changes to the kits during the time frame of the CHMS cycle 1 and the cross-over study analyses. The microbiological assay used at SickKids was specifically modified to mimic that of the NHANES laboratory - using the chloramphenicol-resistant micro-organism (L. rhamnosus) with 5-methyltetrahydrofolate as the calibrator ${ }^{(26)}$. A mean of 12.7 (SD 1.7) ng/ampoule (expected $13 \mathrm{ng} /$ ampoule) and a CV of $13 \%$ were obtained when analysing the 95/528 National Institute for Biological Standards and Control WHO whole-blood reference standard 
over 4 months ( $n 35$ runs). To further verify that the microbiological procedures at SickKids were comparable to those of the NHANES, six quality-control whole-blood haemolysates were analysed blinded at the SickKids laboratory. On average, calculated values were $9 \%$ lower in Toronto compared with the NHANES, with all values within $\pm 20 \%$.

Generation of the cross-over equation. Erythrocyte folate concentrations determined by the microbiological assay and immunoassay were analysed using Analyse-it, version 2.26 Excel $12+$ software (Analyse-it Software Limited). Values were deemed outliers and removed $(n 6)$ from the dataset where the absolute difference in erythrocyte folate concentrations between the two methods for a given blood sample (immunoassay-microbiological assay) was greater than $2 \mathrm{SD}$. Plotting the two sets of the remaining values against each other resulted in a Pearson correlation coefficient $(r)$ of 0.67. We examined the scatter plot of the residuals (standard residual by estimated 'true' value) and determined that curvilinearity was not present. Since the $r$ value was $<0 \cdot 80$, we used Deming regression methods to generate the cross-over equation, as opposed to ordinary least-squares regression which is appropriate when the $r$ value is $\geq 0 \cdot 80$. At an $r$ value of $<0 \cdot 80$, ordinary least-squares regression is known to underestimate the actual slope of the data ${ }^{(33)}$. Furthermore, Deming regression accounts for the random error present in both the values of $X$ and $Y$, whereas ordinary least-squares regression assumes random error for only the $Y$ values, which rarely occurs in methodcomparison studies ${ }^{(34,35)}$. A weighted Deming regression was performed to account for proportional error, or the progressive increase in the scatter of the sample residuals (see online supplementary Fig. S1) ${ }^{(36)}$. Bland-Altman analysis demonstrated a bias of $24 \%$ ( $95 \%$ limits of agreement -26 to $75 \%$ ) between the immunoassay and microbiological assay methods (see online supplementary Fig. S2). The final weighted Deming regression equation was as follows:

Predicted microbiological assay $(\mathrm{nmol} / \mathrm{l})$

$$
=-22.95+0.81 \times \text { immunoassay }(\mathrm{nmol} / \mathrm{l}) \text {. }
$$

We assessed the goodness-of-fit by estimating the mean absolute percentage error (MAPE) resulting from the model. This statistic,

$$
\text { MAPE }=1 / n \sum_{k=1}^{n}\left(\left(y_{k}-\operatorname{pred}_{-} y_{k}\right) / y_{k}\right),
$$

indicated that there was $23 \%$ error in the model, where $k$ is the dummy index or the range for a quantity from $k$ up to, and including, $n ; y$ is the actual value for microbiological assay; pred_y is the predicted value for microbiological assay; and $n$ is the number of fitted points. To validate the model, the study population was randomly split into two groups, one for estimation ( $n$ 76) and the other for validation ( $n$ 76). The estimation sample was used to fit the model, and the validation sample was used to obtain the MAPE. A similar MAPE was obtained, indicating robustness in the precision of the model's predicted values.

As a final examination of the model, we conducted a boundary assessment by generating two additional equations using the lower and upper 95\% CI values of the intercept from the conversion equation. Since the slope was robust, this was held constant. The two additional equations are as follows:

Lower 95\% CI: predicted microbiological assay (nmol/1)

$$
=-155 \cdot 68+0 \cdot 81 \times \text { immunoassay }(\mathrm{nmol} / \mathrm{l}),
$$

Upper 95\% CI: predicted microbiological assay (nmol/l)

$$
=109 \cdot 78+0 \cdot 81 \times \text { immunoassay }(\mathrm{nmol} / \mathrm{l}) .
$$

We applied these equations to the CHMS data to estimate the cumulative distributions at the lower and upper boundaries of the conversion equation.

Since the CHMS immunoassay does not correct for serum in its calculation of erythrocyte folate, and the NHANES microbiological assay does, we also adjusted CHMS whole-blood folate concentrations for comparison with the NHANES whole-blood folate concentrations.

Ethical approval for the cross-over study was obtained from the Children's Hospital of Eastern Ontario, the Hospital for Sick Children, the University of Ottawa and the Health Canada Research Ethics Boards.

\section{Statistical analysis}

Where relevant, each of the analyses was conducted using the unadjusted Canadian erythrocyte folate data first, and then repeated following the application of the cross-over equation to the Canadian erythrocyte folate data to derive a methodadjusted measure for comparison with the NHANES erythrocyte folate data. We stacked the CHMS and NHANES datasets based on regional strata and primary sampling units. Descriptive statistics (frequencies, medians and percentiles) were used to characterise the population. Differences between the countries for erythrocyte folate concentrations and supplement intake by age and race/ethnicity were examined using $t$ tests. Differences between the countries for whole-blood folate concentrations were also examined using $t$ tests. All estimates were based on weighted data to represent the population. Variance estimation $(95 \% \mathrm{CI})$ and significance testing ( $t$ tests and logistic regression) were based on Taylor linearisation ${ }^{(37)}$. Significance was defined as a $P$ value of $<0 \cdot 05$. Analyses were conducted in SAS 9.1.3 (SAS Institute, Inc.) and SUDAAN version 10.0 (RTI International, Research Triangle Park).

\section{Results}

A summary of the demographics of the study populations and folic acid supplement use is given in Table 1. It shows that $25 \%$ of the general population in Canada reported folic acid supplement use, whereas in the USA this was $32 \%$.

\section{Unadjusted erythrocyte folate}

Less than $1 \%$ of the population in Canada or the USA had clinical folate deficiency (erythrocyte folate concentration $<305 \mathrm{nmol} / \mathrm{l}$ ). The median (50th percentile) of the unadjusted Canadian erythrocyte folate data $(1250 \mathrm{nmol} / \mathrm{l})$ was higher 
Table 1. Summary description of population demographics and folic acid supplement use by country

(Percentages and $95 \%$ confidence intervals)

\begin{tabular}{|c|c|c|c|c|c|c|}
\hline & \multicolumn{6}{|c|}{ General population } \\
\hline & \multicolumn{3}{|c|}{ Canada ( $n$ 5248) } & \multicolumn{3}{|c|}{ USA $(n 7070)$} \\
\hline & \multirow[b]{2}{*}{ Sample size $(n)$} & \multicolumn{2}{|c|}{$\begin{array}{l}\text { Weighted } \\
\text { distribution }\end{array}$} & \multirow[b]{2}{*}{ Sample size $(n)$} & \multicolumn{2}{|c|}{$\begin{array}{l}\text { Weighted } \\
\text { distribution }\end{array}$} \\
\hline & & $\%$ & $95 \% \mathrm{Cl}$ & & $\%$ & $95 \% \mathrm{Cl}$ \\
\hline \multicolumn{7}{|l|}{ Sociodemographic factors } \\
\hline \multicolumn{7}{|l|}{ Age (years) } \\
\hline $6-11$ & 911 & $6 \cdot 6$ & $5 \cdot 9,7 \cdot 3$ & 1010 & 8.0 & $7 \cdot 4,8 \cdot 7$ \\
\hline $12-19$ & 945 & $11 \cdot 1$ & $10 \cdot 0,12 \cdot 3$ & 1073 & $12 \cdot 2$ & $11 \cdot 0,13 \cdot 4$ \\
\hline $20-39$ & 1150 & $31 \cdot 3$ & $27 \cdot 6,35 \cdot 2$ & 1705 & $30 \cdot 7$ & $28 \cdot 5,33 \cdot 1$ \\
\hline $40-59$ & 1202 & $34 \cdot 2$ & $31 \cdot 9,36 \cdot 6$ & 1722 & $32 \cdot 9$ & $31 \cdot 1,34 \cdot 7$ \\
\hline $60-79$ & 1040 & $16 \cdot 8$ & $13 \cdot 8,20 \cdot 3$ & 1560 & $16 \cdot 2$ & $14 \cdot 4,18 \cdot 3$ \\
\hline \multicolumn{7}{|l|}{ Sex } \\
\hline Female & 2705 & $49 \cdot 8$ & $46 \cdot 7,52 \cdot 9$ & 3546 & $50 \cdot 7$ & $49 \cdot 7,51 \cdot 7$ \\
\hline Male & 2543 & $50 \cdot 2$ & $47 \cdot 1,53 \cdot 3$ & 3524 & $49 \cdot 3$ & $48 \cdot 3,50 \cdot 3$ \\
\hline \multicolumn{7}{|l|}{ Income quintile } \\
\hline Q1 & 1125 & 18.5 & $15 \cdot 2,22 \cdot 3$ & 1908 & $17 \cdot 6$ & $14 \cdot 8,20 \cdot 8$ \\
\hline Q2 & 1130 & $20 \cdot 8$ & $18 \cdot 3,23 \cdot 6$ & 1625 & $18 \cdot 4$ & $15 \cdot 9,21 \cdot 1$ \\
\hline Q3 & 838 & $16 \cdot 8$ & $14 \cdot 8,19 \cdot 1$ & 1384 & $20 \cdot 7$ & $18 \cdot 8,22 \cdot 8$ \\
\hline Q4 & 980 & $18 \cdot 8$ & $15 \cdot 9,22 \cdot 1$ & 905 & $20 \cdot 1$ & $17 \cdot 0,23 \cdot 8$ \\
\hline Q5 & 843 & $18 \cdot 3$ & $14 \cdot 9,22 \cdot 4$ & 726 & $16 \cdot 7$ & $14 \cdot 6,19 \cdot 1$ \\
\hline Missing & 332 & $6 \cdot 8$ & $5 \cdot 9,7 \cdot 8$ & 522 & $6 \cdot 4$ & $4 \cdot 9,8 \cdot 3$ \\
\hline \multicolumn{7}{|l|}{ Race/ethnicity } \\
\hline Non-Hispanic white & 4499 & $84 \cdot 6$ & $73 \cdot 8,91 \cdot 4$ & 4155 & $67 \cdot 3$ & $59 \cdot 4,74 \cdot 4$ \\
\hline Other & 749 & $15 \cdot 4^{*}$ & $8 \cdot 6,26 \cdot 2$ & 2915 & $32 \cdot 7$ & $25 \cdot 6,40 \cdot 6$ \\
\hline \multicolumn{7}{|l|}{ Supplement use } \\
\hline \multicolumn{7}{|l|}{ Folic acid supplement (last $30 \mathrm{~d}$ ) } \\
\hline Yes (at least one) & 1258 & $24 \cdot 6$ & $22 \cdot 3,27 \cdot 0$ & 1873 & $32 \cdot 0$ & $28 \cdot 7,35 \cdot 5$ \\
\hline No & 3990 & $75 \cdot 4$ & $73 \cdot 0,77 \cdot 7$ & 5197 & $68 \cdot 0$ & $64 \cdot 5,71 \cdot 3$ \\
\hline \multicolumn{7}{|c|}{ Strength of folic acid supplement (total dose) } \\
\hline $0 \mu \mathrm{g}(\mathrm{did}$ not take in the last $30 \mathrm{~d})$ & 3990 & $75 \cdot 4$ & $73 \cdot 0,77 \cdot 7$ & 5197 & $68 \cdot 0$ & $64 \cdot 5,71 \cdot 3$ \\
\hline $1-399 \mu \mathrm{g}$ & 496 & 7.5 & $6 \cdot 5,8 \cdot 6$ & 847 & $13 \cdot 8$ & $12 \cdot 3,15 \cdot 4$ \\
\hline $400-999 \mu \mathrm{g}$ & 568 & $13 \cdot 0$ & $11 \cdot 1,15 \cdot 2$ & 976 & $17 \cdot 3$ & $15 \cdot 4,19 \cdot 5$ \\
\hline$\geq 1000 \mu \mathrm{g}$ & 194 & $4 \cdot 1$ & $3 \cdot 3,5 \cdot 0$ & 50 & $0 \cdot 8^{\star}$ & $0 \cdot 6,1 \cdot 2$ \\
\hline
\end{tabular}

* Interpret with caution (high sampling variability; CV $\geq 16.6$ and $<33 \cdot 3$ ).

than the American median of $1100 \mathrm{nmol} / \mathrm{l}$ (Table 2). Median erythrocyte folate concentrations were significantly higher for Canadians compared with Americans among folic acid supplement users and non-users, for a race/ethnicity other than non-Hispanic white, and for individuals aged 12-59 years (Table 3).

\section{Method-adjusted erythrocyte folate}

Consistent with the unadjusted analyses, less than $1 \%$ of the population in Canada or the USA were folate deficient following adjustment of CHMS erythrocyte folate values (Fig. 1). In contrast to the unadjusted analyses, the methodadjusted median erythrocyte folate concentration of Canadians was significantly lower than the median value for Americans (988 v. $1100 \mathrm{nmol} / 1$, respectively; Table 2). This significant difference persisted for the youngest age groups (6-19 years) and the oldest age group (60-79 years), as well as for folic acid supplement users and non-Hispanic whites (Table 3). Comparing the method-adjusted Canadian whole-blood folate concentration in comparison to the American whole-blood folate distribution (see online supplementary Table S1) - to

Table 2. Selected percentiles for erythrocyte folate concentrations by country $\dagger$

(Percentiles and $95 \%$ confidence intervals)

\begin{tabular}{|c|c|c|c|c|c|c|c|c|c|c|c|c|}
\hline & $\begin{array}{c}\text { 5th } \\
\text { percentile }\end{array}$ & $95 \% \mathrm{Cl}$ & $\begin{array}{c}\text { 25th } \\
\text { percentile }\end{array}$ & $95 \% \mathrm{Cl}$ & $\begin{array}{l}\text { 50th } \\
\text { percentile }\end{array}$ & $95 \% \mathrm{Cl}$ & $\begin{array}{c}\text { 75th } \\
\text { percentile }\end{array}$ & $95 \% \mathrm{Cl}$ & $\begin{array}{c}\text { 95th } \\
\text { percentile }\end{array}$ & $95 \% \mathrm{Cl}$ & $\begin{array}{c}\text { 97th } \\
\text { percentile }\end{array}$ & $95 \% \mathrm{Cl}$ \\
\hline \multicolumn{13}{|c|}{ General population (nmol/l) } \\
\hline $\begin{array}{l}\text { Canada } \\
\quad \text { (unadjusted) }\end{array}$ & 621 & 569,714 & 960 & 886,1040 & $1250^{*}$ & 1170,1320 & 1620 & 1500,1710 & 2316 & 2170,2490 & 2580 & 2350,2740 \\
\hline $\begin{array}{l}\text { Canada } \\
\text { (method-adjusted) }\end{array}$ & 480 & 438,556 & 755 & 695,817 & $988^{*}$ & 925,1050 & 1290 & 1200,1360 & 1850 & 1730,1990 & 2070 & 1880,2200 \\
\hline USA & 565 & 545,591 & 852 & 817,893 & 1100 & 1050,1160 & 1440 & 1370,1510 & 2150 & 1990,2260 & 2370 & 2250,2490 \\
\hline
\end{tabular}

${ }^{*} P<0.05$ for tests comparing the unadjusted and method-adjusted Canadian concentrations at the 50th percentile with the US distribution.

$\dagger$ Tests were not conducted at the 5th, 25th, 75th, 95th and 97th percentiles. 
Table 3. Erythrocyte folate for country by age, race/ethnicity and folic acid supplement use for the general population (Medians and $95 \%$ confidence intervals)

\begin{tabular}{|c|c|c|c|c|c|c|}
\hline & \multicolumn{2}{|c|}{$\begin{array}{l}\text { Canada (unadjusted) } \\
\text { (nmol/l) }\end{array}$} & \multicolumn{2}{|c|}{$\begin{array}{l}\text { Canada (method- } \\
\text { adjusted) (nmol/l) }\end{array}$} & \multicolumn{2}{|c|}{ USA (nmol/I) } \\
\hline & Median & $95 \% \mathrm{Cl}$ & Median & $95 \% \mathrm{Cl}$ & Median & $95 \% \mathrm{Cl}$ \\
\hline \multicolumn{7}{|l|}{ General population } \\
\hline \multicolumn{7}{|l|}{ Age (years) } \\
\hline $6-11$ & 1210 & 1140,1300 & 959 & 902,1030 & 1130 & 1100,1190 \\
\hline $12-19$ & $1120^{*}$ & 1000,1190 & $884^{*}$ & 788,941 & 974 & 933,1040 \\
\hline $20-39$ & $1190^{*}$ & 1100,1250 & 941 & 871,993 & 1010 & 976,1070 \\
\hline $40-59$ & $1290^{*}$ & 1180,1440 & 1020 & 933,1140 & 1150 & 1070,1220 \\
\hline $60-79$ & 1410 & 1310,1540 & $1120^{*}$ & 1040,1220 & 1420 & 1310,1530 \\
\hline \multicolumn{7}{|l|}{ Race/ethnicity } \\
\hline Non-Hispanic white & 1260 & 1170,1340 & $994^{*}$ & 926,1060 & 1170 & 1110,1240 \\
\hline Other & $1190^{*}$ & 1130,1300 & 941 & 893,1030 & 980 & 930,1030 \\
\hline \multicolumn{7}{|c|}{ Folic acid supplement use (last $30 \mathrm{~d}$ ) } \\
\hline Yes (at least one) & $1590^{*}$ & 1480,1700 & $1260^{\star}$ & 1180,1350 & 1370 & 1330,1440 \\
\hline No & $1180^{*}$ & 1090,1250 & 930 & 861,993 & 998 & 964,1048 \\
\hline
\end{tabular}

understand the impact of correcting erythrocyte folate for the contribution of serum folate, which the NHANES does, but the CHMS does not - demonstrated similar parallel distributions across both countries as observed with the erythrocyte folate distributions (Table 2), suggesting that the lack of correction for the contribution of serum folate in the CHMS was not a likely reason for the observed differences between the two countries.

\section{Boundary assessment}

The examination of the extreme boundaries of the conversion equation demonstrated that the cumulative distribution of erythrocyte folate concentrations in the upper boundary for the method-adjusted Canadian data did not approach the unadjusted Canadian values, except at the 5 th percentile (see online supplementary Fig. S3). When comparing the boundaries for the method-adjusted Canadian data with the USA, the cumulative distributions overlapped at the upper boundary, while the lower boundary was below the US distribution.

\section{Discussion}

The present study presents the first national-level comparison of folate status based on erythrocyte concentrations between Canada and the USA. To facilitate these analyses, we generated a conversion equation to account for the different analytical methods employed to measure erythrocyte folate concentration at the national level in the two countries. The fact that erythrocyte folate concentrations were either higher or lower in Canadians compared with Americans, depending on whether an adjustment was made to account for assay differences, suggests that caution must be exercised in evaluating erythrocyte folate data from different countries because analytical methods are not readily comparable. Furthermore, we cannot unequivocally conclude that there are true differences in erythrocyte folate concentrations between the Canadian and American populations in the post-fortification era.
Comparing the distribution of erythrocyte folate concentrations for Canadians and Americans is of interest from the perspective of population-level surveillance. Assessing these distributions, particularly in countries where folic acid fortification and supplementation policies are in place, provides information on the success of these interventions and indicates where there may be a need for modification. The virtual absence of clinical deficiency demonstrates the success of folic acid-related public health efforts in both countries, despite certain differences. It is notable that both countries demonstrate median folate concentrations that are much higher than the deficiency cut-off of $305 \mathrm{nmol} / \mathrm{l}$. We observed an apparent difference in median erythrocyte folate status between Canada and the USA; however, the upper boundary of the method-adjusted CHMS distribution overlapped that of the NHANES distribution, and the lower boundary was considerably below that of the NHANES distribution. Further investigation into the presence of a disparity between countries, and factors driving a potential difference, is needed. In the present study, a smaller proportion of Canadians reported taking folic acid supplements, a primary predictor

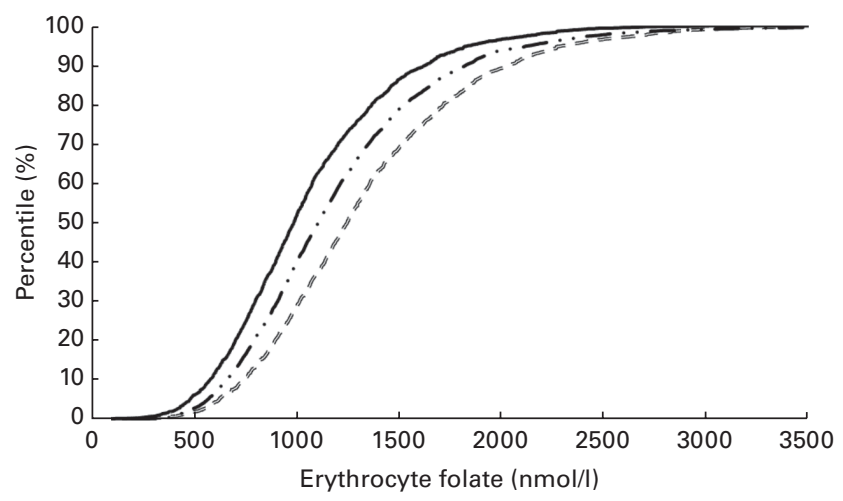

Fig. 1. Cumulative percentile distributions of erythrocyte folate concentrations by country from the 2007-9 Canadian Health Measures Survey (adjusted $(-)$ for microbiological assay comparison and unadjusted $(=)$ ) and the 2007-8 National Health and Nutrition Examination Survey (USA, - .). Values exceeding $3500 \mathrm{nmol} / \mathrm{l}$ are not shown ( $n$ 32). 
of folate status, than Americans ${ }^{(38)}$. The proportions of folic acid supplement consumption for each country in the present study are congruent with those reported in past examinations of nationally representative Canadian and American data ${ }^{(39,40)}$. Dietary intake of folic acid may also be a factor since fortification policies differ in some aspects between the two countries. Most significantly, the USA mandates a greater number of foods to be fortified and Canada allows lower fortification levels for breakfast cereals ${ }^{(1,2)}$. The limited CHMS dietary data precluded our ability to examine dietary intake as a factor in the discrepancy between countries.

There is growing support for harmonisation of biochemical measurement methods in national-level studies. With the change in the NHANES erythrocyte folate concentration measurement method from the Bio-Rad radioassay (before 2006) to the microbiological assay (2006-10), there came the need to formulate regression equations to allow for accurate comparisons of erythrocyte folate data across survey periods ${ }^{(41)}$. Adjustment of CHMS erythrocyte folate concentrations using our calibration equation resulted in different conclusions regarding median erythrocyte folate concentrations between the two countries. The median unadjusted Canadian erythrocyte folate concentration of $1250 \mathrm{nmol} / \mathrm{l}$ was higher than the American median of $1100 \mathrm{nmol} / \mathrm{l}$, whereas the method-adjusted Canadian value of $988 \mathrm{nmol} / 1$ was lower. Vitamin D is an example of a nutrient currently undergoing methodological scrutiny as numerous and varying measurement methods have impeded the ability to formulate evidence-based guidelines and surveillance efforts ${ }^{(42)}$. It has been suggested that standardised measures are needed to determine appropriate levels in the population that are comparable to other countries. Similarly, harmonising folate measurements between Canada and the USA would allow for effective monitoring and assessment of the longitudinal trajectory of elevated levels to determine the need for modification to current programmes. While harmonisation of survey data may be achievable through well-designed comparison studies that generate a conversion equation between two methods, standardisation can only be pursued when standard reference materials and higher-order reference measurement procedures are available. This is not yet the case for erythrocyte folate.

The present study is the first comparison of erythrocyte folate concentrations between two nationally representative North American datasets using harmonised data. Although it is imperative to harmonise methods before making comparisons, method-comparison studies are not without limitations. The CHMS data are limited as the manufacturer's serum and whole-blood controls were used to assess the accuracy and reproducibility of the assay, rather than an external standard reference material as per the NHANES methodology. A certain level of error is evident in our conversion equation, as indicated by the relatively poor correlation and wide CI demonstrated in the boundary assessment. Our examination of method-adjusted whole-blood folate concentration provides assurance that not correcting for serum folate concentrations in the CHMS immunoassay - where the NHANES microbiological assay does - had no influence on the direction or magnitude of our findings. Since the microbiological assay for the CHMS method comparison was not conducted at the NHANES laboratory, we examined the agreement between the SickKids laboratory and NHANES microbiological assays based on a small set of six blood samples, and found that the results were within $\pm 20 \%$ of each other, which is fairly common for an assay that has an imprecision of approximately $10 \%$. On average, the SickKids laboratory microbiological assay was $9 \%$ lower than the NHANES microbiological assay, thus the study may be limited by a potential underestimation of the adjusted CHMS erythrocyte folate values. Considering the low correlation, and potential interlaboratory and inter-assay variation in erythrocyte folate measurement, caution should be used in applying the conversion equation outside of the current context.

Comparing erythrocyte folate data across time and countries is an important step in evaluating the success of current interventions, and will advance future dialogue and research on folic acid fortification and supplementation policies. The present study highlights that caution must be exercised in comparing erythrocyte folate data from different countries since the use of analytical methods that have not been harmonised may lead to incorrect data interpretation, and, furthermore, we do not recommend that the conversion equation be applied outside of the current context. Adjusting laboratory data allows for a comparison of erythrocyte folate data; however, it is important to consider the limitations of the method-comparison study. Harmonisation of folate measurement methods in future surveillance efforts would facilitate comparisons and inform policy directions.

\section{Supplementary material}

To view supplementary material for this article, please visit http://dx.doi.org/10.1017/S0007114514002906

\section{Acknowledgements}

The authors thank the Health Analysis Division at Statistics Canada, in particular Julie Bernier for her support during the generation of the conversion equation, and the Physical Health Measures Division at Statistics Canada for preparing and granting special access to data. The authors also thank Steve Brooks and Penny Jee for the CHMS laboratory blood analysis in the conversion equation sub-study and Trevor Stewart for his review and feedback on the manuscript. The authors thank all participants in the conversion equation sub-study, the CHMS and the NHANES. This work was supported by Canadian Institutes of Health Research (CIHR) Health Professionals Fellowship in the Area of Public Health (funding reference no. 180375) to C.K.C. and a CIHR Operating Grant (funding reference no. 218776) to M. S. T., D. L. O. and C. K. C.

The authors' contributions to the study were as follows: C. K. C., M. S. T. and D. L. O. conceptualised and designed the study and crossover equation sub-study. S. A. and D. L. O. conducted the data collection and blood analysis for the sub-study. C. K. C. generated the crossover equation with 
the assistance of T. B., M. S. T., C. M. P. and D. L. O. C. K. C. conducted all analyses and T. B., M. S. T., C. M. P. and D. L. O. assisted in the interpretation of the results. C. K. C. drafted the manuscript. All of the authors read and approved the final manuscript.

The findings and conclusions in this report are those of the authors and do not necessarily represent the official views or positions of the Centers for Disease Control and Prevention/ Agency for Toxic Substances and Disease Registry.

\section{References}

1. Food and Drug Administration (1996) Food standards: amendment of standards of identity for enriched grain products to require addition of folic acid. Final rule. 21 CFR Parts 136, 137, and 139. Fed Regist 61, 8781-8789.

2. Canada Gazette (1996) Food and drug regulations - amendment SOR/96-527. Canada Gazette Part II. http://publications. gc.ca/gazette/archives/p2/1998/1998-11-25/pdf/g2-13224.pdf (accessed March 2014).

3. Berry RJ, Mulinare J \& Hamner H (2010) Folic acid fortification: neural tube defect risk reduction - a global perspective. In Folate in Health and Disease, 2nd ed., pp. 179-204 [LB Bailey, editor]. Boca Raton, FL: CRC Press, Taylor \& Francis Group.

4. Czeizel AE \& Dudas I (1992) Prevention of the first occurrence of neural-tube defects by periconceptional vitamin supplementation. N Engl J Med 327, 1832-1835.

5. MRC Vitamin Study Research Group (1991) Prevention of neural tube defects: results of the Medical Research Council Vitamin Study. Lancet 338, 131-137.

6. De Wals P, Tairou F, Van Allen MI, et al. (2007) Reduction in neural-tube defects after folic acid fortification in Canada. $N$ Engl J Med 357, 135-142.

7. Williams LJ, Rasmussen SA, Flores A, et al. (2005) Decline in the prevalence of spina bifida and anencephaly by race/ ethnicity: 1995-2002. Pediatrics 116, 580-586.

8. Centers for Disease Control and Prevention (CDC) (2010) CDC Grand Rounds: additional opportunities to prevent neural tube defects with folic acid fortification. MMWR Morb Mortal Wkly Rep 59, 980-984.

9. Flour Fortification Initiative (2012) Global Progress Mandatory Wheat Flour Fortification Legislation - August 2012. Ottawa, ON: Flours Fortification Initiative. http://www. ffinetwork.org/global_progress/index.php (accessed 11 December 2012).

10. Crider KS, Bailey LB \& Berry RJ (2011) Folic acid food fortification - its history, effect, concerns, and future directions. Nutrients 3, 370-384.

11. Institute of Medicine (1998) Dietary Reference Intakes for Thiamin, Riboflavin, Niacin, Vitamin $B_{6}$, Folate, Vitamin $B_{12}$ Pantothenic Acid, Biotin, and Choline. Washington, DC: National Academy Press.

12. Health Canada (2014) Prenatal Nutrition Guidelines for Health Professionals - Folate Contributes to a Healthy Pregnancy. Ottawa, ON: Health Canada. http://www.hc-sc.gc.ca/ fn-an/pubs/nutrition/folate-eng.php (accessed March 2014).

13. Yetley EA \& Johnson CL (2011) Folate and vitamin B-12 biomarkers in NHANES: history of their measurement and use. Am J Clin Nutr 94, 322S-331S.

14. Colapinto CK, O'Connor DL \& Tremblay MS (2011) Folate status of the population in the Canadian health measures survey. CMAJ 183, E100-E106.
15. Pfeiffer CM, Hughes JP, Lacher DA, et al. (2012) Estimation of trends in serum and RBC folate in the U.S. population from pre- to postfortification using assay-adjusted data from the NHANES 1988-2010. J Nutr 142, 886-893.

16. Dietrich M, Brown CJ \& Block G (2005) The effect of folate fortification of cereal-grain products on blood folate status, dietary folate intake, and dietary folate sources among adult non-supplement users in the United States. J Am Coll Nutr 24, 266-274.

17. Gunter EW, Bowman BA, Caudill SP, et al. (1996) Results of an international round robin for serum and whole-blood folate. Clin Chem 42, 1689-1694.

18. Owen WE \& Roberts WL (2003) Comparison of five automated serum and whole blood folate assays. Am J Clin Pathol 120, 121-126.

19. Fazili Z, Pfeiffer CM, Zhang M, et al. (2008) Influence of 5,10-methylenetetrahydrofolate reductase polymorphism on whole-blood folate concentrations measured by LC-MS/ MS, microbiologic assay, and Bio-Rad radioassay. Clin Chem 54, 197-201.

20. Pfeiffer CM, Zhang M, Lacher DA, et al. (2011) Comparison of serum and red blood cell folate microbiologic assays for national population surveys. J Nutr 141, 1402-1409.

21. Icke GC, Dennis M, Sjollema S, et al. (2004) Red cell $N^{5}$-methyltetrahydrofolate concentrations and C677T methylenetetrahydrofolate reductase genotype in patients with stroke. J Clin Pathol 57, 54-57.

22. Bryan S, St-Denis M \& Wojtas D (2007) Canadian Health Measures Survey: clinic operations and logistics. Health Rep 18, Suppl., 53-70.

23. Centres for Disease Control and Prevention (2014) About the National Health and Nutrition Examination Survey. Washington, DC: Centres for Disease Control and Prevention. http://www.cdc.gov/nchs/nhanes/about_nhanes.htm (accessed 13 May 2013).

24. Day B, Langlois R \& Tremblay M (2007) Canadian Health Measures Survey: ethical, legal and social issues. Health Rep 18, Suppl., 37-51.

25. McNulty B, Pentieva K, Marshall B, et al. (2011) Women's compliance with current folic acid recommendations and achievement of optimal vitamin status for preventing neural tube defects. Hum Reprod 26, 1530-1536.

26. Centres for Disease Control and Prevention (2008) Laboratory procedure manual: folate. http://www.cdc.gov/nchs/ data/nhanes/nhanes_07_08/FOLATE_E_met.pdf (accessed 22 September 2012).

27. Siemens Canada Limited (2009) Immulite 2000 Folic Acid Insert. Mississauga, ON: Siemens Canada Limited.

28. Centres for Disease Control and Prevention (2011) National Health and Nutrition Examination Survey: 2007-2008, Data Documentation, Codebook, and Frequencies: RBC Folate and Serum Folate. Washington, DC: Centres for Disease Control and Prevention. http://www.cdc.gov/ nchs/nhanes/nhanes2007-2008/FOLATE_E.htm\#LBXRBFSI. (accessed 13 May 2013).

29. Selhub J, Jacques PF, Dallal G, et al. (2008) The use of blood concentrations of vitamins and their respective functional indicators to define folate and vitamin $\mathrm{B}_{12}$ status. Food Nutr Bull 29, S67-S73.

30. Statistics Canada (2007) Questionnaire(s) and Reporting Guide(s) - Canadian Health Measures Survey (CHMS). Ottawa, ON: Statistics Canada. http://www23.statcan.gc.ca/ $\mathrm{imdb} / \mathrm{p} 2$ SV.pl?Function $=$ getSurvInstrument\&SurvId=9353\& InstaId=10263 (accessed 10 June 2014). 
31. Health Canada (2013) Drug Product Database. Ottawa, ON: Health Canada. http://www.hc-sc.gc.ca/dhp-mps/prodpharma/ databasdon/index-eng.php (accessed 13 May 2013).

32. Health Canada (2014) Licensed Natural Health Products Database. Ottawa, ON: Health Canada. http://www.hc-sc. gc.ca.proxy.bib.uottawa.ca/dhp-mps/prodnatur/applications/ licen-prod/lnhpd-bdpsnh_data_extract-eng.php (accessed 13 May 2013).

33. Good PI \& Hardin JW (2009) Common Errors in Statistics (and How to Avoid Them). Hoboken, NJ: John Wiley and Sons, Inc.

34. Ludbrook J (2002) Statistical techniques for comparing measurers and methods of measurement: a critical review. Clin Exp Pharmacol Physiol 29, 527-536.

35. Linnet K (1993) Evaluation of regression procedures for methods comparison studies. Clin Chem 39, 424-432.

36. Linnet K (1990) Estimation of the linear relationship between the measurements of two methods with proportional errors. Stat Med 9, 1463-1473.

37. Rust K \& Rao J (1996) Variance estimation for complex surveys using replication techniques. Stat Methods Med Res $\mathbf{5}, 281-310$
38. Colapinto CK, O'Connor DL, Dubois L, et al. (2012) Folic acid supplement use is the most significant predictor of folate concentrations in Canadian women of childbearing age. Appl Physiol Nutr Metab 37, 284-292.

39. Shakur YA, Garriguet D, Corey P, et al. (2010) Folic acid fortification above mandated levels results in a low prevalence of folate inadequacy among Canadians. Am J Clin Nutr 92, 818-825.

40. Tinker SC, Cogswell ME, Devine O, et al. (2010) Folic acid intake among U.S. women aged 15-44 years, National Health and Nutrition Examination Survey, 2003-2006. Am J Prev Med 38, 534-542.

41. Pfeiffer CM, Hughes JP, Durazo-Arvizu RA, et al. (2012) Changes in measurement procedure from a radioassay to a microbiologic assay necessitate adjustment of serum and RBC folate concentrations in the U.S. population from the NHANES 1988-2010. J Nutr 142, 894-900.

42. Sempos CT, Vesper HW, Phinney KW, et al. (2012) Vitamin D status as an international issue: national surveys and the problem of standardization. Scand J Clin Lab Invest Suppl 243, 32-40. 